\title{
Primary care physician perceptions of non-steroidal anti-inflammatory drug and aspirin-associated toxicity: results of a national survey
}

W. D. CHEY*, S. ESWAREN*, C. W. HOWDEN†, J. M. INADOMI*, A. M. FENDRICK\$ \& J. M. SCHEIMAN*

*Division of Gastroenterology, University of Michigan, Ann Arbor, MI; $\dagger$ Division of Gastroenterology, Northwestern University Feinberg School of Medicine, Chicago, IL; $\$$ Division of General Internal Medicine, University of Michigan, Ann Arbor, MI, USA

Correspondence to:

Dr W. D. Chey, University of

Michigan Medical Center, 3912

Taubman Center, PO Box 0362, Ann

Arbor, MI 48109, USA.

E-mail: wchey@umich.edu

\section{Publication data}

Submitted 6 October 2005

First decision 17 October 2005

Resubmitted 9 November 2005

Resubmitted 15 December 2005

Accepted 16 December 2005

\section{SUMMARY}

Aim

To assess primary care physician perceptions of non-steroidal antiinflammatory drug (NSAID) and aspirin-associated toxicity.

\section{Methods}

A group of gastroenterologists and internal medicine physicians created a survey, which was administered via the Internet to a large number of primary care physicians from across the US.

\section{Results}

One thousand primary care physicians participated. Almost one-third of primary care physicians recommended $325 \mathrm{mg}$ rather than $81 \mathrm{mg}$ of aspirin/day for cardioprotection. Fifty-nine percent thought enteric-coated or buffered aspirin reduced the risk of upper gastrointestinal (GI) bleeding. Seventy-six percent believed that Helicobacter pylori infection increased the risk of NSAID ulcers but fewer than 25\% tested NSAID users for this infection. More than two-thirds were aware that aspirin co-therapy decreased the GI safety benefits of the cyclo-oxygenase 2 selective NSAIDs. However, 84\% felt that aspirin with a cyclo-oxygenase 2 selective NSAID was safer than aspirin with a non-selective NSAID. When presented a patient at high risk for NSAID-related GI toxicity, almost 50\% of primary care physicians recommended a proton pump inhibitor and cyclo-oxygenase 2 selective NSAID.

\section{Conclusions}

This survey has identified areas of misinformation regarding the riskbenefit of NSAIDs and aspirin and the utilization of gastroprotective strategies. Further education on NSAIDs for primary care physicians is warranted. 


\section{INTRODUCTION}

There is a considerable morbidity and mortality associated with non-steroidal anti-inflammatory drug (NSAID)-related gastrointestinal injury. ${ }^{1}$ Based upon data from the US, over 111 million prescriptions for anti-inflammatory drugs were written in 2004, with additional patient exposure in the over-the-counter (OTC) NSAID market, translating to an enormous cohort at risk. ${ }^{2}$

Estimates of as many as 107000 hospitalizations and 16500 deaths result from NSAID-induced serious adverse events in the US alone. ${ }^{3}$ These untoward events are of importance not only in terms of the resultant morbidity and mortality; but also such complications incur substantial resource use. ${ }^{4}$

Primary care physicians (PCPs) prescribe a substantial proportion of NSAIDs. However, dissemination and incorporation of new information in this rapidly evolving field can be slow and is subject to the influence of promotional efforts initiated by the pharmaceutical industry, which not only target providers but have aggressively marketed innovations in this area directly to consumers.

Non-steroidal anti-inflammatory drugs are inhibitors of cyclo-oxygenase (COX), which has at least two isoforms: cyclo-oxygenase $1(\mathrm{COX}-1)$ and cyclo-oxygenase 2 (COX-2). Traditional 'non-selective' NSAIDs and aspirin antagonize the actions of both COX-1 and -2. Their depletion of gastrointestinal mucosal prostaglandins explains their gastrointestinal toxicity. The COX-2-selective NSAIDs have been shown in large outcome studies to be associated with lower rates of upper and lower gastrointestinal (GI) adverse events than non-selective NSAIDs ${ }^{5}$ - but only among patients who do not concomitantly use aspirin. ${ }^{6-7}$

The efficacy of non-selective and COX-2-selective NSAIDs in treating pain and inflammation should be considered in concert with their recognized gastrointestinal toxicity and increasingly, with consideration of their cardiovascular risk. $^{8-9}$ The gastrointestinal risks - at least those in the upper GI tract - can be reduced by the concomitant prescription of a 'gastroprotective' agent such as a proton pump inhibitor (PPI) or misoprostol. ${ }^{10-11}$

To better understand the perceptions of the potential risks associated with the use of aspirin, non-selective and COX-2-selective NSAIDs, and the use of gastroprotective strategies, we conducted a survey research study amongst a large, geographically diverse group of PCPs from the US. Our data were collected late in 2003 prior to the withdrawal of rofecoxib and valdecoxib. Although a great deal of attention has subsequently been focused on the cardiovascular risk associated with the COX-2-selective NSAIDs, a number of other important issues regarding the use of aspirin, NSAIDs and gastroprotective medications remain. The results of this survey serve to identify a number of areas which require further clarification and education.

\section{METHODS}

The questionnaire was developed by the investigators without influence from the pharmaceutical sponsor. The items elicited information regarding PCP practices and perceptions on aspirin, NSAID and gastroprotective medication use.

\section{Study population}

A PCP was defined as a general practitioner, family practitioner or internal medicine physician. Three thousand, eight hundred and sixteen PCPs from a panel provided by a professional Internet survey research organization (Ziment, New York, NY, USA) were invited to participate. Potential study participants were solicited by e-mail. Those that opted to participate were asked several screening questions to be sure that they were appropriate for the survey. In order to participate, PCPs had to have been practising medicine in the US for at least two but no more than 35 years, to spend no more than $50 \%$ of their time in a teaching capacity, and to write at least 15 prescriptions per month for the management of pain or inflammation. Exclusion criteria included working for (or having an immediate family member who worked for) an advertising agency, marketing company, pharmaceutical company or news organization. Those that did not meet the screening criteria were not allowed to complete the survey.

A geographically diverse cohort of 1000 PCPs completed the on-line survey anonymously from November 20 through December 8, 2003. Solicitation was closed after 1000 completed questionnaires were obtained. A fee of $\$ 25$ was offered for completing the survey. Funding for the services provided by the survey research organization and participant honoraria 
were provided by TAP Pharmaceuticals (Chicago, IL, USA).

\section{Survey administration}

The order of questions presented in the survey can be found in the Appendix. Participants could only proceed to the next question after completing the question currently on their computer monitor. Once a question was completed and the participant proceeded to the next question, they could not go back and change an answer to a previous question. Participants were neither encouraged nor discouraged to use reference materials when completing the survey.

\section{Questionnaire content}

\section{Aspirin issues}

Questions probed beliefs regarding the efficacy of aspirin as a primary or secondary prevention strategy for myocardial infarction (MI) and stroke as well as prescribing behaviours (recommended dose and frequency of prescribing) of aspirin for those indications. Participants were asked if enteric-coated or buffered aspirin reduced the likelihood of developing an upper GI-bleeding event compared with regular aspirin.

A hypothetical situation was posed involving a patient with a history of an ulcer-related upper GI bleed that required low-dose aspirin for a history of coronary artery disease. Participants were asked to choose between continuing the aspirin without any change in therapy, switching to enteric-coated aspirin, adding an $\mathrm{H}_{2}$-receptor antagonist $\left(\mathrm{H}_{2} \mathrm{RA}\right)$ in a dose available for OTC purchase, adding an $\mathrm{H}_{2} \mathrm{RA}$ at prescription strength, adding a PPI, or adding misoprostol.

\section{Utilization of non-selective NSAIDs and associated risks}

The questionnaire queried about how often elderly patients (>70 years) used NSAIDs, the type of NSAID an individual PCP most often prescribed, and the gastrointestinal risks of COX-2-specific agents compared with non-selective NSAIDs. Participants were asked their belief on whether the presence of dyspeptic symptoms identified patients more likely to develop an NSAID-associated ulcer complication and whether non-selective NSAIDs are associated with an increased risk of bleeding from the small intestine and/or colon.

\section{NSAIDs and Helicobacter pylori infection}

To asses the level of understanding of the relationship between NSAIDs and H. pylori, we asked if the presence of $H$. pylori would reduce, increase or have no net effect on the likelihood of ulcers in NSAID users. Participants were also asked about the frequency of testing for $H$. pylori in patients taking NSAIDs.

\section{Utilization and safety of COX-2-selective NSAIDs}

Participants were asked if COX-2-selective NSAIDs reduced the likelihood of significant adverse GI events such as bleeding, perforation or obstruction compared with non-selective NSAIDs. They were also asked if the GI safety benefits of the COX-2-selective NSAIDs were maintained in patients concurrently taking lowdose aspirin. Beliefs about the possibility of increased cardiovascular risks associated with rofecoxib were also assessed. We asked PCPs if they felt that rofecoxib was associated with an increased risk of MI and if the potential for adverse cardiac effects influenced their decision to prescribe this medication.

\section{Hypothetical patient scenarios}

To better understand the strategies employed by PCPs to reduce the risk of an NSAID-associated GI complication, a series of hypothetical patient scenarios were constructed. These patient scenarios were designed to assess how PCPs apply their knowledge of the risks and benefits of aspirin, NSAIDs and gastroprotective medications in clinical practice situations. PCPs were asked what they would recommend for the following patient scenarios:

(i) 'A patient, with a recently healed H. pylori negative, NSAID-associated ulcer, who requires an NSAID for joint pain'. Choices offered included change to a COX-2-selective NSAID, add a PPI, change to a COX2-selective NSAID and add a PPI, add misoprostol, add a $\mathrm{H}_{2} \mathrm{RA}$, add sucralfate or use an alternative traditional NSAID and a PPI.

(ii) 'A patient, with a history of previous MI, who requires low-dose aspirin for cardioprotection and an NSAID for arthritis related pain'. Options offered to study participants included aspirin and a traditional NSAID; aspirin with a traditional NSAID and PPI; aspirin with a traditional NSAID and misoprostol; aspirin with a traditional NSAID and a $\mathrm{H}_{2} \mathrm{RA}$; aspirin with a COX-2-selective NSAID; or aspirin with a COX2-selective NSAID and PPI. 
(iii) 'A patient, with a past history of an ulcer-related upper gastrointestinal bleed, who needs to be on an NSAID for joint pain and also needs low-dose aspirin for a history of coronary artery disease'. For this question, PCPs chose from aspirin with a traditional NSAID; aspirin with a traditional NSAID and PPI; aspirin with a traditional NSAID and misoprostol; aspirin with a traditional NSAID and $\mathrm{H}_{2} \mathrm{RA}$; aspirin with a COX-2-selective NSAID; or aspirin with a COX2-selective NSAID and PPI.

\section{Statistical analysis}

Descriptive statistics characterized the responses to the questionnaire.

Comparisons between subgroups of the study sample (gender-, age- and region-based comparisons) were performed using the chi-squared test (QUANTUM software; SPSS, Chicago, IL, USA). In situations where answers to a question created a continuous variable, a Student's $t$-test was utilized. A $P$-value of less than 0.05 defined a statistically significant difference between values. When present, significant differences in the data based upon the gender, age, and region of residence are mentioned in the results section.

\section{RESULTS}

\section{Demographics}

Table 1 summarizes the demographic data for the 1000 respondents. Eighty percent of respondents were male. A discussion of how our sample compared with PCPs at large and the limitations of our survey methodology can be found in the Discussion section. Most PCPs reported insurance coverage for their patients: 24\% Health Maintenance Organization, 20\% Preferred Provider Organization, 8\% Point of Service, 27\% Medicare and 8\% Medicaid.

\section{Aspirin issues}

Consistent with the recently published US Preventive Services Task Force document, ninety-one percent of PCPs felt that aspirin had been shown to be effective in the primary prevention of MI. ${ }^{12}$ Though the available evidence would suggest otherwise, $85 \%$ felt that aspirin had been shown to be of benefit for the primary prevention of stroke. ${ }^{12}$ Sixty-two percent of participants reported that they always recommended aspirin for the primary prevention of MI. Ninety-five percent recommended aspirin for primary prevention of MI always or most of the time ( $>50 \%$ of the time). Ninety-one percent recommended aspirin all or most of the time for the primary prevention of stroke. These recommendations were not influenced by the gender, age or region of residence of the respondents. Nearly a third recommended aspirin $325 \mathrm{mg}$ daily rather than $81 \mathrm{mg}$ daily for the primary prevention of MI (Figure 1). PCPs under the age of 55 years recommended the higher dose of aspirin more commonly than PCPs over the age of 55 years $(32 \%$ vs. $20 \%, P=0.0035)$. For their patients requiring aspirin for cardioprotection, 59\% of participants thought enteric-coated or buffered aspirin reduced the risk of serious upper GI-bleeding events despite a lack of evidence to support this belief. More female than male PCPs believed that enteric coating or buffering of aspirin reduced the risk of developing a serious gastrointestinal bleeding event (67\% vs. 57\%, $P=0.0094$ ). When presented a patient with known cardiac disease and a history of previous upper gastrointestinal bleeding but no current dyspeptic symptoms, 53\% of participants recommended switching from non-coated to enteric-coated aspirin while only $26 \%$ recommended the addition of a PPI. Perhaps more disturbingly, 9\% recommended no change in therapy. The remaining 12\% suggested the addition of an OTC or prescription $\mathrm{H}_{2} \mathrm{RA}$ or misoprostol.

\section{Utilization of non-selective NSAIDs and associated risks}

Fifty percent of participants reported that over half of their patients older than age 70 years used an NSAID

\begin{tabular}{lr}
\hline Table 1. Demographic data & \\
\hline Completed surveys & 1000 \\
General practitioners & 54 \\
Family practitioners & 465 \\
Internal medicine physicians & 481 \\
Physician age (\%) & \\
Under 35 & 10 \\
35-44 & 35 \\
45-54 & 38 \\
55-64 & 14 \\
65 or older & 1 \\
Physician gender (\%) & \\
Male & 80 \\
Female & 20
\end{tabular}




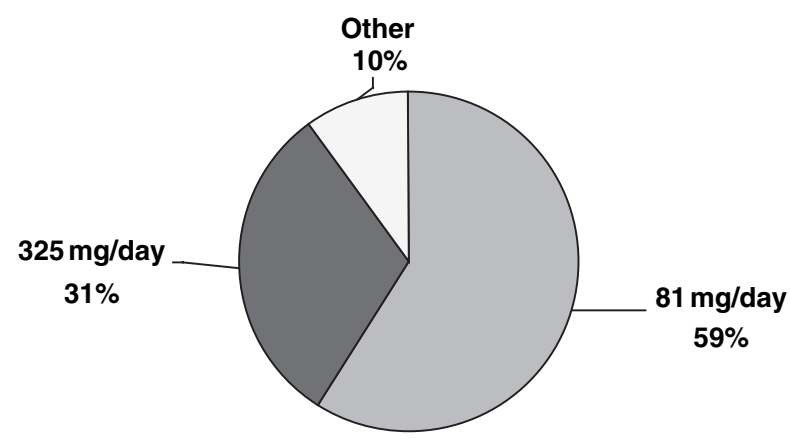

Figure 1. Dose of aspirin recommended by primary care physicians for cardioprotection.

at least once per week while $42 \%$ said that the correct percentage of weekly NSAID users was between 30\% and 50\%. Participants reported 57\% of their NSAID prescriptions were for a non-selective agent while 43\% of their NSAID prescriptions were for a COX-2-selective NSAID. Younger PCPs were less likely than older PCPs to recommend a COX-2-selective NSAID than a traditional NSAID ( $42 \%$ vs. $47 \%, P=0.005)$. PCPs from the west coast (37\%) were less likely to prescribe a COX-2-selective NSAID than their counterparts from the northeast (42\%), south $(44 \%)$ or central states $(45 \%)$.

Despite data to the contrary, ${ }^{13-14}$ more than a third (34\%) believed that most patients who developed upper gastrointestinal bleeding while taking NSAID experienced antecedent dyspeptic symptoms. Seventysix percent of participants were aware that NSAIDs increased the risk of bleeding from the small intestine and/or colon. Responses were similar regardless of the gender, age or region of residence of the respondents.

\section{NSAIDs and $H$. pylori infection}

Seventy-six percent of participants believed that the presence of $H$. pylori infection increased the likelihood of an NSAID-associated ulcer while 19\% felt that H. pylori played no role. Three percent actually felt that $H$. pylori decreased the risk of an NSAID-associated ulcer. PCPs over the age of 55 years were more likely than those $<55$ years to believe that $H$. pylori increased the likelihood of ulcers in NSAID users ( $88 \%$ vs. $76 \%, P=0.0009$ ). Though the majority of PCPs felt that $H$. pylori increased the risk of developing an NSAID-associated ulcer, fewer than a quarter recommended testing for this infection in patients initiating or already taking NSAID therapy. Opinions on testing for H. pylori before starting an NSAID or in those patients already on an NSAID are presented in Figure 2. Responses to questions in this section of the questionnaire were not influenced by the gender or residence of the respondents.

\section{Utilization and safety of COX-2-selective NSAIDs}

Ninety-four percent of participants believed that COX2-selective agents reduced the likelihood of adverse GI events compared to non-selective NSAIDs. Sixtyfour percent of the PCPs were aware that co-therapy with aspirin decreased the GI safety benefits offered by the COX-2-selective NSAIDs while $26 \%$ felt that aspirin had no effect and 5\% actually felt that aspirin improved the GI safety benefits of these agents. A greater percentage of older than younger PCPs believed that low-dose aspirin had no effect on the gastrointestinal safety benefits of a COX-2-selective NSAID (33\% vs. $24 \%, P=0.0317$ ). However, despite a lack of evidence, an overwhelming majority (84\%) felt that aspirin combined with a COX-2-selective NSAID was safer than aspirin combined with a non-selective NSAID. Only 6\% of PCPs felt that rofecoxib was associated with a higher likelihood of MI than other COX-2-selective NSAIDs while 57\% felt that the cardiovascular risk for rofecoxib was no greater than for other COX-2-selective NSAIDs. Twelve percent reported that concern over cardiovascular safety with rofecoxib influenced their prescribing behaviour while 48\% stated that such concerns did not influence their prescribing of rofecoxib. Gender, age or residence of PCP did not influence the responses.

\section{Hypothetical patient scenarios}

Figures 3-5 detail the respondents' recommendations for the three hypothetical patient scenarios described in the methods section. As seen in Figure 3 nearly half of PCPs recommended the combination of a COXselective NSAID and a PPI in a patient with recent NSAID-associated ulcer bleeding. Approximately a fifth of respondents recommended either adding a PPI to a non-selective NSAID or using a COX-2-selective NSAID alone in this patient.

For the patient with cardiac disease in need of antiplatelet therapy and an NSAID for joint pain (Figure 4), just under half of the respondents recommended the 

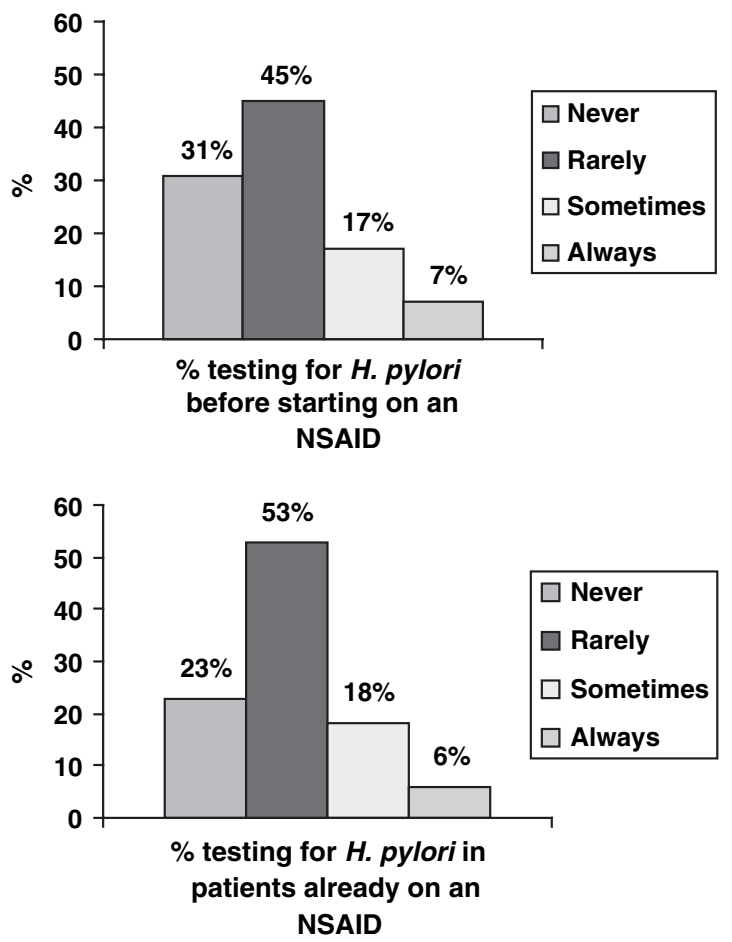

Figure 2. Respondents' use of Helicobacter pylori testing in non-steroidal anti-inflammatory drug users.

combination of aspirin and a COX-2-selective NSAID while over a quarter recommended aspirin with a nonselective NSAID. Physicians over the age of 55 more commonly recommended aspirin and a COX-2-selective NSAID than those under the age of 55 years (57\% vs. $43 \%, P=0.0014)$. Fourteen percent recommended aspirin with a COX-2-selective NSAID and PPI. Only 9\% selected the combination of aspirin, non-selective NSAID and PPI.

Finally, for the patient with a recent ulcer bleed who requires antiplatelet therapy for cardioprotection and an NSAID for joint pain (Figure 5), 60\% chose aspirin, a COX-2-selective NSAID and a PPI. Somewhat disturbingly, a quarter selected the combination of aspirin and a COX-2-selective NSAID. Only 9\% selected aspirin, a non-selective NSAID and a PPI.

\section{DISCUSSION}

Our survey identified some significant gaps in knowledge regarding the risks and benefits of aspirin and NSAID therapy. Further, this data raises some interesting questions regarding the utilization of gastroprotective therapy in patients using aspirin or NSAIDs.

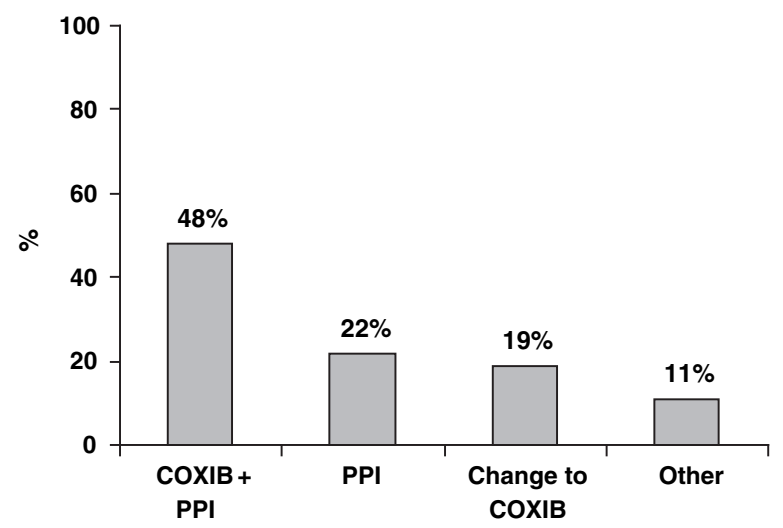

Figure 3. Recommended changes for a patient with a recent ulcer-related upper GI bleed who needs an NSAID for joint pain. Asa $=$ Aspirin, $\mathrm{COXIB}=\mathrm{COX} 2$ selective NSAID, PPI = proton pump inhibitor.

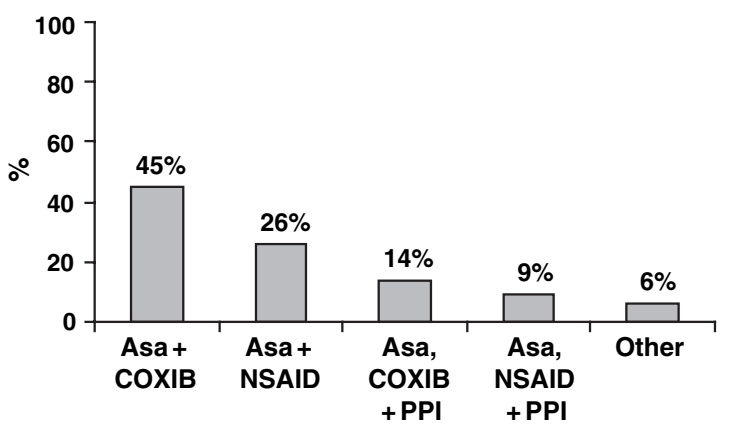

Figure 4. Recommended changes for a patient with no history of ulcer bleeding who needs aspirin for cardioprotection and an NSAID for joint pain. Asa = Aspirin, $\mathrm{COXIB}=\mathrm{COX} 2$ selective NSAID, PPI $=$ proton pump inhibitor.

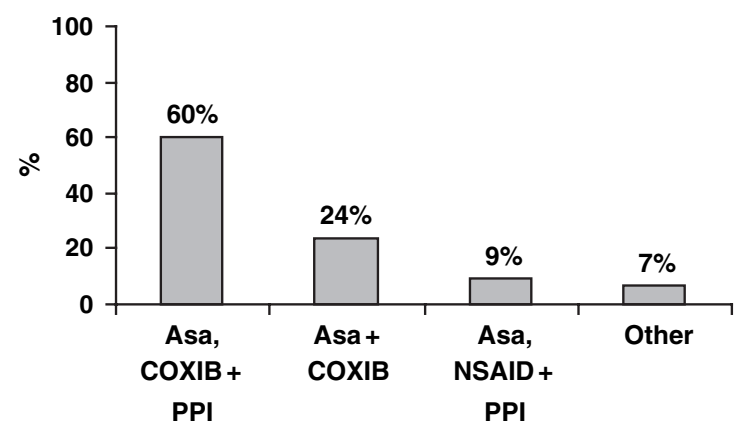

Figure 5. Recommended changes for a patient with a history of a previous ulcer-related upper GI bleed who needs aspirin for cardioprotection and an NSAID for joint pain. Asa $=$ Aspirin, COXIB $=$ COX 2 selective NSAID, PPI $=$ proton pump inhibitor. 
Aspirin use, either with of without the advice of a physician, is increasing in the US. ${ }^{15}$ The potential risks associated with the use of low-dose aspirin appear to be under-recognized by both consumers and healthcare providers. Although a dose as low as $75 \mathrm{mg}$ per day has been shown to offer cardioproctective benefit, ${ }^{12,16}$ many consumers take larger doses, presumably in the belief that benefit is dose-related and because the higher dose has a lower acquisition cost. Unfortunately, this may increase the GI risk without adding additional cardiovascular benefit. ${ }^{17}$ To this point, nearly one-third of our primary care participants recommended daily doses of aspirin greater than $81 \mathrm{mg}$ per day for cardioprotection. Further, a significant percentage of PCPs reported that they typically recommended aspirin for primary prevention of cardiac events in all patients. Although antiplatelet therapy, particularly aspirin, has been found to significantly reduce recurrent cardiovascular and vascular events (secondary prevention - those with a prior cardiovascular or vascular event), its role in primary prophylaxis (those with no history of a cardiovascular or vascular event) remains the subject of some debate. ${ }^{12,16}$ It is clear that the benefits of aspirin must be weighed against the potential problems that such treatment can induce. Even low doses of aspirin can have detrimental effects on the gastric mucosa. When combined with the antiplatelet effects of aspirin, it is not surprising that a meta-analysis of 24 randomized trials involving over 66000 patients reported a 2 -fold increase in the risk of gastrointestinal bleeding for those taking chronic low-dose aspirin compared to those not taking aspirin. ${ }^{18}$ In addition, aspirin use may be associated with an increased risk of haemorrhagic stroke. These well-documented safety concerns led the US Preventative Services Task Force to recommend that a risk-benefit calculation be performed before recommending low-dose aspirin for primary prevention of cardiovascular events. The Task Force concluded that the balance of risk and benefit of aspirin was strongly tied to cardiac risk, explicitly recommending prophylactic aspirin only to those with a 3\% or greater five year risk of cardiovascular events. Calculation of such risk is easily performed using Personal Digital Assistant or Internet based tools. ${ }^{12,16}$

Surprisingly, fewer than one-third of PCPs recommended an accepted gastroprotective agent for aspirin users at high risk for a GI complication. This is concerning as a recent trial from Hong Kong ${ }^{19}$ reported that patients with a history of aspirin-associated ulcer bleeding who were continued on low-dose aspirin without a gastroprotective agent had a one year ulcer rebleeding risk of approximately 15\%. On the other hand, the addition of a PPI to aspirin was associated with a significant reduction in the annual recurrence of ulcer rebleeding. Further, more than half (53\%) of our survey respondents recommended switching from regular aspirin to enteric coated aspirin rather than adding a gastroprotective agent in patients with a history of an aspirin-associated UGI bleed who require cardioprotection. Available data suggests that entericcoating or buffering of aspirin does not reduce the risk of upper GI bleeding. ${ }^{18,20}$ To date, there have been no placebo-controlled, randomized trials with misoprostol or histamine-2-recpetor antagonists in high risk patients taking aspirin.

Nearly one quarter of participants was unaware of the deleterious effects that aspirin exerts on the GI safety benefits of the COX-2-selective NSAIDs. ${ }^{6-7,21}$ Eightyfour percent thought that adverse GI events would be less likely with the combination of low-dose aspirin and a COX-2-selective NSAID compared to low-dose aspirin and a non-selective NSAID. While there are no prospective randomized controlled trials which have addressed this issue as a primary endpoint, the results of secondary analyses and database studies suggest that aspirin with a COX-2-selective NSAID are associated with a similar risk of ulcer and upper GI bleeding as a non-selective NSAID. ${ }^{22}$ Despite these deleterious effects of aspirin on the GI safety benefits of the COX-2-selective NSAIDs, $24 \%$ of our participants recommended the combination of aspirin and a COX-2-selective NSAID for a hypothetical patient with GI risk factors - without the addition of a gastroprotective agent (Figure 5).

With regard to $H$. pylori infection in patients taking an NSAID, 78\% of PCPs believed there was a relationship between H. pylori and the presence of NSAID associated ulcers. While there appears to be an additive risk of complications in infected individuals using an NSAID, ${ }^{23} 76 \%$ of practitioners never or rarely tested for the presence of $H$. pylori before starting a NSAID. A similar percentage of PCPs reported that they never or rarely tested for the presence of $H$. pylori in those already taking chronic NSAID therapy. This likely reflects several issues including the lack of any formal recommendation to address $H$. pylori in NSAID users from professional organizations in the US, conflicting data regarding the benefits of treating $H$. pylori in NSAID users, ${ }^{24-27}$ and the complexity of testing and eradication regimens for $H$. pylori infection. ${ }^{28}$ 
In this survey which was performed prior to the removal of 2 of the 3 available COX-2-selective NSAIDs from the US market, PCPs appeared to use COX-2-selective NSAIDs as first line therapy. In fact, PCPs reported that $43 \%$ of their NSAID prescriptions were for the COX-2-selective NSAIDs. This is somewhat curious given modeling studies which have clearly demonstrated that these agents are only cost-effective in patients at higher risk for an NSAID associated gastrointestinal complication. ${ }^{29-30}$ At the time of our survey, less than 5\% of PCPs felt that rofecoxib was associated with a greater risk of MI than other COX-2selective NSAIDs and only 12\% reported that these concerns significantly influenced their decision to prescribe rofecoxib. These findings are fascinating given the reports of an increased incidence of MI with rofecoxib dating back to publication of the VIGOR trial in $2000^{5}$ and subsequently raised by other analyses prior to the time of our survey. ${ }^{31}$

The hypothetical patient scenarios also warrant comment. At the time of our survey, many PCPs were recommending the use of a COX-2-selective NSAID with a PPI in patients at high risk for an NSAID related complication. Though there was data to suggest that the combination of a PPI and non-selective NSAID or a COX-2-selective NSAID alone was associated with a lower rate of gastrointestinal complications than a non-selective NSAID alone, there was no data to suggest an incremental benefit to combining a PPI and a COX-2-selective NSAID in late 2003. Subsequent data suggest that patients with a previous ulcer bleed who receive a non-selective NSAID and PPI or COX-2-selective NSAID alone may have a 6 month ulcer recurrence and/or rebleeding rate as high of 24$32 \% .{ }^{11}$ Given this high rate of recurrent complications and recently published economic data, ${ }^{32}$ it appears reasonable to suggest the combination of a COX-2selective NSAID and PPI in such high risk patients. In the 2 scenarios where a patient required an aspirin for cardioprotection and an NSAID for joint pain, it is interesting that such a high percentage of PCPs recommended aspirin with a COX-2-selective NSAID, despite the fact that more than two-thirds understood that aspirin decreased or eliminated the GI safety benefits of a COX-2-selective NSAID. This highlights the distressing fact that factual knowledge does not always translate into clinical practice. It is not clear how recent events surrounding the COX-2-selective NSAIDs have affected prescribing patterns amongst PCPs though one would speculate that utilization of gastro- protective medications is likely to have replaced at least some of the use of the COX-2-selective NSAIDs in high risk patients.

The greatest strength of this study is the large, nationally representative sample of physicians that participated. As with any survey, our study has a number of limitations. We contacted a large group of PCPs by e-mail, which likely biased our study population toward younger PCPs with access to and who regularly used e-mail. Our demographic data confirms this as only $1 \%$ of our participants were over the age of 65 years. Amongst members of the American Medical Association (not stratified by specialty), 18\% were over the age of 65 years. We cannot say for certain how this may have affected our results. However, we would argue that younger physicians who are held to recently imposed recertification standards are likely to be more up to date on new medical findings than their older colleagues. We also note that $80 \%$ of our study cohort was male. This is similar to data from the AMA in 2000 which reported that $76 \%$ of its membership was male.${ }^{33}$ Unfortunately, we have no demographic information about those who chose not to participate. It is possible that our participants were more interested in the subject of the survey than those who did not participate. However if that were the case, our results should represent the views of relatively well-informed PCPs in the US and thus makes the results even more compelling. The results reflect doctors' self-reported practice patterns, practices that are not verified by chart reviews. Doctors may either overestimate or underestimate their practices depending on behaviours that might be perceived as incorrect or not standard of care. Another point worthy of consideration is that we did not encourage or discourage PCPs to use resource materials when completing the survey. We feel this reflects real life where PCPs can readily access reference materials if a question arises in their clinical practice. Though there is no way to definitively know whether or not PCPs utilized resource materials, we were able to gauge how long it took individual respondents to complete the survey. Presumably, those taking an excessive amount of time to complete the survey may have been more likely to have utilized reference materials. We have reviewed this data and time to complete the survey fell within a very tight range with no obvious outliers. As such, we do not feel that the use of reference materials adversely affected the results of our survey. Further, and perhaps most importantly, if PCPs had utilized reference materials, 
one would expect an artificially increased reporting of 'correct' answers to survey questions and that is not what we found.

We would also point out that there is no flawless way to select a participant pool for a survey study. Using a mass mailing to the membership of organizations such as the American Medical Association (AMA) or American College of Physicians (ACP) may not be truly representative of PCPs. For example, surveying members of the ACP would capture primarily internists and would largely ignore Family Medicine physicians and General Practitioners. Further, survey research studies which rely on mass mailings are typically associated with poor response rates raising issues of generalizability of results.

Given the recent withdrawal of two COX-2-selective NSAIDs from the US market because of adverse cardiovascular events, a better understanding regarding approaches to reducing ulcer risk has greater importance than ever for physicians treating patients in need of an NSAID or aspirin. Our survey has uncovered a number of important areas of misinformation regarding this complex treatment area which requires an appropriate understanding of the benefits and risks associated with these commonly used agents. Identifying and addressing the existing knowledge deficits offers the opportunity to reduce adverse events and improve outcomes in patients who require an NSAID and/or aspirin.

\section{ACKNOWLEDGEMENTS}

Funding for the services provided by the survey research organisation and participant honoraria were provided by TAP Pharmaceuticals (Chicago, IL, USA).

\section{REFERENCES}

1 Del Valle J, Chey WD, Scheiman JM. Chapter 66: Acid-peptic disorders. In: Yamada, T, ed. Textbook of Gastroenterology, 4th edn. Philadelphia, PA: JM Lippincott, 2003: 1321-76.

2 IMS Health. National Prescription Audit Plus $^{T M}$, 2004 (January 2004-December 2004).

3 Wolfe MM, Lichtenstein DR, Singh G. Gastrointestinal toxicity of nonsteroidal antiinflammatory drugs. $N$ Engl J Med 1999; 340: 1888-99.

4 Peloso PM, Scheiman JM. The economic implications of COX-2 specific inhibitors. Am J Med 2001; 110: 50S-4S.

5 Bombardier C, Laine L, Reicin A, et al. Comparison of upper gastrointestinal toxicity of rofecoxib and naproxen in patients with rheumatoid arthritis. $N$ Engl J Med 2000; 343: 1520-8.

6 Silverstein FE, Faich G, Goldstein JL, et al. Gastrointestinal toxicity with celecoxib vs nonsteroidal anti-inflammatory drugs for osteoarthritis and rheumatoid arthritis. JAMA 2000; 284: 1247-55.

7 Goldstein JL, Eisen GM, Agrawal N, et al. Reduced incidence of upper gastrointestinal ulcer complications with the COX-2 selective inhibitor, valdecoxib. Aliment Pharmacol Ther 2004; 20: 527-38.

8 Hippisley-Cox J, Coupland C. Risk of myocardial infarction in patients taking cyclo-oxygenase-2 inhibitors or conventional non-steroidal anti-inflammatory drugs: population based nested cast-control analysis. $\mathrm{Br}$ Med J 2005; 330: 1366-9.

9 Lévesque LE, Brophy JM, Zhang B. The risk of myocardial infarction with cyclooxygenase-2 inhibitors: a population study of elderly adults. Ann Intern Med 2005; 142: 481-9.

10 Graham DY, Agrawal NM, Campbell DR, et al. Ulcer prevention in long-term users of nonsteroidal anti-inflammatory drugs. Arch Intern Med 2002; 162: 169-75.

11 Chan FKL, Hung LCT, Suen BY, et al. Celecoxib versus diclofenac plus omeprazole in high-risk arthritis patients: results of a randomized double-blind trial. Gastroenterology 2004; 127: 1038-43.

12 U.S. Preventive Services Task Force. Aspirin for the primary prevention of cardiovascular events: recommendation and rationale. Ann Intern Med 2002; 136: 157-60.

13 Armstrong CP, Blower AL. Non-steroidal anti-inflammatory drugs and life threatening complications of peptic ulceration. Gut 1987; 28: 527-32.

14 Singh G, Ramey DR, Morfield D, et al. Gastrointestinal tract complications of nonsteroidal anti-inflammatory drug treatment in rheumatoid arthritis. A prospective observational cohort study. Arch Intern Med 1996; 156: 1530-6.
15 Scheiman JM, Fendrick AM. NSAIDs without a prescription: over-counter access, under counted risks. Am J Gastroenterol 2002; 97: 2159-60.

16 Hayden M, Pignone M, Phillips C, et al. Aspirin for the primary prevention of cardiovascular events: a summary of the evidence for the U.S. preventive services task force. Ann Intern Med 2002; 136: 161-72.

17 Weil J, Colin-Jones D, Langman M, et al. Prophylactic aspirin and risk of peptic ulcer bleeding. Br Med J 1995; 310: 827-30.

18 Derry S, Loke YK. Risk of gastrointestinal haemorrhage with long term use of aspirin: meta-analysis. $\mathrm{Br}$ Med $\mathrm{J} 2000$; 321: 1183-7.

19 Lai KC, Lam SK, Chu KM, et al. Lansoprazole for the prevention of recurrences of ulcer complications from long-term low-dose aspirin use. $N$ Engl $\mathrm{J} \mathrm{Med}$ 2002; 346: 2033-88.

20 Kelly JP, Kaufman DW, Jurgelon JM, et al. Risk of aspirin-associated major upper-gastrointestinal bleeding with enteric-coated or buffered product. Lancet 1996; 348: 1413-6.

21 Laine L, Maller ES, Yu C, et al. Ulcer formation with low-dose enteric-coated aspirin and the effect of COX-2 selective inhibition: a double-blind trial. Gastroenterology 2004; 127: 395-402.

22 Lanas A, Garcia-Rodriguez LA, Arroyo MT, et al. Coxibs, NSAIDs, Aspirin, PPIs 
and the risks of upper GI bleeding in common clinical practice. Gastroenterology 2005; 128: A-94 (abstract 629).

23 Huang JQ, Sridhar S, Hunt RH. Role of Helicobacter pylori infection and nonsteroidal anti-inflammatory drugs in peptic-ulcer disease: a meta-analysis. Lancet 2002; 359: 14-22.

24 Campbell DR, Haber MM, Sheldon E, et al. Effect of $H$. pylori status on gastric ulcer healing in patients continuing nonsteroidal anti-inflammatory therapy and receiving treatment with lansoprazole or ranitidine. Am J Gastroenterol 2002; 97: 2208-14.

25 Hawkey CJ, Næsdal J, Wilson I, et al. Relative contribution of mucosal injury and Helicobacter pylori in the development of gastroduodenal lesions in patients taking non-steroidal antiinflammatory drugs. Gut 2002; 51: 336-43.
26 Chan FKL, To KF, Wu JCY, et al. Eradication of Helicobacter pylori and risk of peptic ulcers in patients starting longterm treatment with non-steroidal antiinflammatory drugs: a randomized trial. Lancet 2002; 359: 9-13.

27 Lai KC, Lam SK, Chu CM, et al. Lansoprazole reduces ulcer relapse after eradication of Helicobacter pylori in nonsteroidal anti-inflammatory drug users - a randomized trial. Aliment Pharmacol Ther 2003; 18: 829-36.

28 Saad R, Chey WD. A clinician's guide to the diagnosis and treatment of $H$. pylori. Clev Clin J Med 2005; 72: 109-26.

29 Fendrick AM, Bandekar RR, Chernew $\mathrm{ME}$, et al. Role of initial NSAID choice and patient risk factors in the prevention of NSAID gastropathy: a decision analysis. Arthritis Care Res 2002; 47: 36-43.
30 Spiegel BMR, Targownik L, Dulai GS, et al. The cost-effectiveness of cyclooxygenase-2 selective inhibitors in the management of chronic arthritis. Ann Intern Med 2003; 138: 795-806.

31 Mukherjee D, Nissen SE, Topol EJ. Risk of cardiovascular events associated with selective COX-2 inhibitors. JAMA 2001; 286: 954-9.

32 Lanas A. Economic analysis of strategies in the prevention of non-steroidal antiinflammatory drug-induced complications in the gastrointestinal tract. Aliment Pharmacol Ther 2004; 20: 321-31.

33 American Medical Association. Physicians in the United States and Possessions by Selected Characteristics. Chicago: c. 1995-2004 [updated 2004 Jun 24; cited 2004 Nov. 12]. Available at: http://www.ama-assn.org/ama/pub/ category/2688.html 
NSAID USAGE ANALYSIS STUDY

\section{Main questionnaire}

\section{Treatment Behaviours}

See Appendix for product and terminology chart. this chart will be shown to all physicians at the start of the main questionnaire.

1. How often do you recommend aspirin as primary preventive therapy for:

\begin{tabular}{lllll}
\hline & Always & Most of the time ( $>50 \%$ of time) & Sometimes $(<50 \%$ of time) & Never \\
\hline $\begin{array}{c}\text { Myocardial infarction } \\
\text { (MI) in subjects }\end{array}$ & 4 & 3 & 2 & 1 \\
$\begin{array}{c}\text { over the age of } 60 \\
\begin{array}{c}\text { Stroke in subjects } \\
\text { over the age of } 60\end{array}\end{array}$ & 4 & 3 & 2 & 1 \\
\hline
\end{tabular}

2. When you recommend the use of aspirin for patients with cardiac disease, what dose do you typically recommend?

$325 \mathrm{mg}$ per day
$325 \mathrm{mg}$ every other day
$81 \mathrm{mg}$ per day
$81 \mathrm{mg}$ every other day $\ldots \ldots \ldots \ldots \ldots \ldots$

3. For a patient with a history of an ulcer-related upper GI bleed in the past but has no current GI symptoms who needs to be on low-dose aspirin for a history of coronary artery disease, what would you most likely recommend?

Continue the aspirin without change in therapy .............................................................................. 1

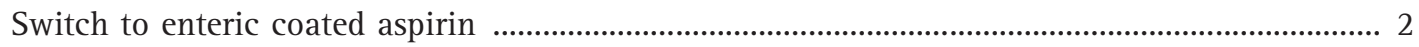

Add an OTC strength H2RA for symptoms only ………............................................................... 3

Add prescription strength H2RA therapy ………….......................................................................... 4

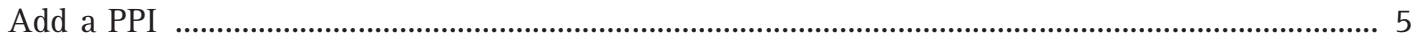

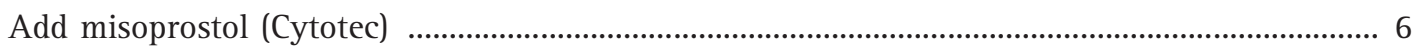

4. When treating a patient with H. pylori-negative, NSAID-associated gastric ulcer, which do you typically recommend?

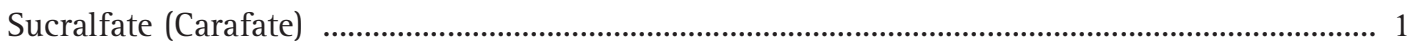

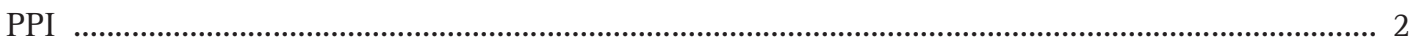

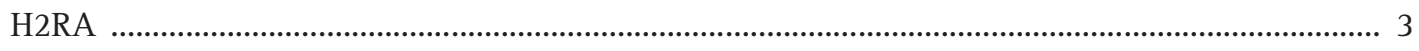

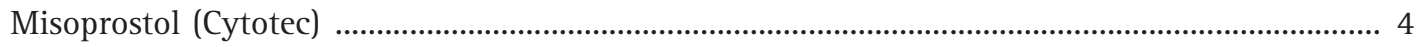

5. When treating a patient with $H$. pylori-negative, NSAID-associated duodenal ulcer, which do you typically recommend?

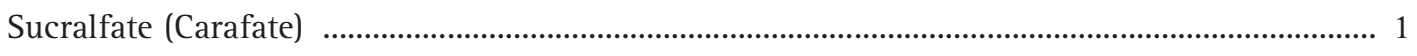

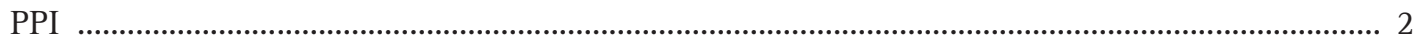

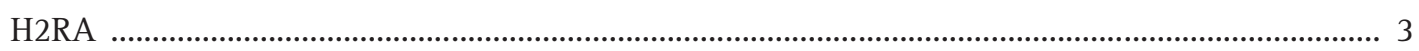

Misoprostol (Cytotec) ...................................................................................................................... 4

6. When attempting to reduce the likelihood of recurrent ulcer related bleeding in a patient with a recently healed H. pylori negative, NSAID-associated ulcer, who requires the NSAID for joint pain, which would you most likely recommend (assuming the patient was on a traditional NSAID)?

Add sucralfate (Carafate) 1 
Add a PPI

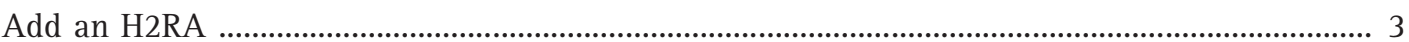

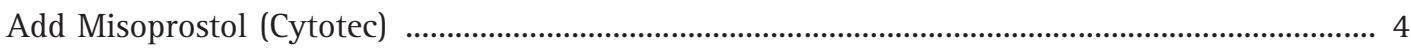

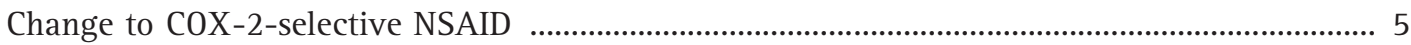

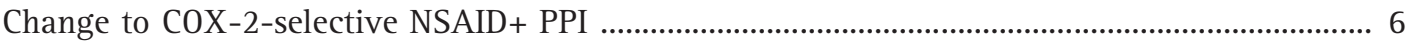

Change to an alternate traditional NSAID + PPI ................................................................ 7

7. In a subject with a history of previous MI who requires low-dose aspirin for cardioprotection and an NSAID for arthritis related pain, which would you most likely recommend (assuming the subject has no other risk factors for an NSAID associated GI complication)?

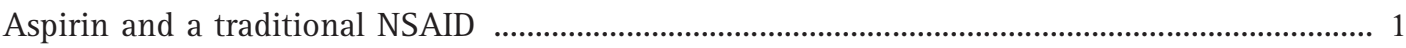

Aspirin and a traditional NSAID with an H2RA .................................................................... 2

Aspirin and a traditional NSAID with a PPI ........................................................................... 3

Aspirin and a traditional NSAID with misoprostol (Cytotec) ..................................................... 4

Aspirin and a COX-2-selective agent (instead of the traditional NSAID) ................................. 5

Aspirin and a COX-2-selective agent (instead of the traditional NSAID) with a PPI ................ 6

8. In a patient with a past history of an ulcer-related upper GI bleed who needs to be on an NSAID for joint pain and also needs low-dose aspirin for a history of coronary artery disease, what would you most likely recommend to reduce the likelihood of recurrent ulcer bleeding?

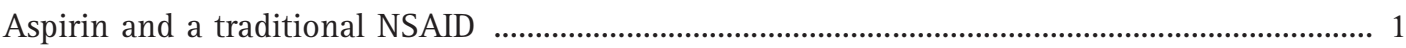

Aspirin and a traditional NSAID with an H2RA .................................................................... 2

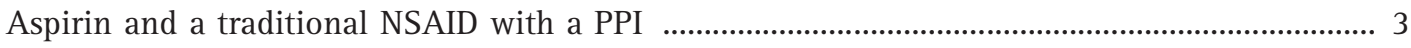

Aspirin and a traditional NSAID with misoprostol (Cytotec) ...................................................... 4

Aspirin and a COX-2-selective agent (instead of the traditional NSAID) .................................. 5

Aspirin and a COX-2-selective agent (instead of the traditional NSAID) with a PPI ................. 6

9. How often do you test for H. pylori in patients starting on an NSAID?

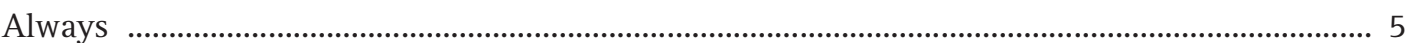

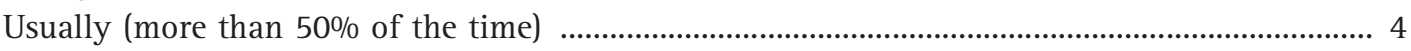

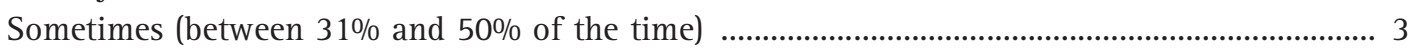

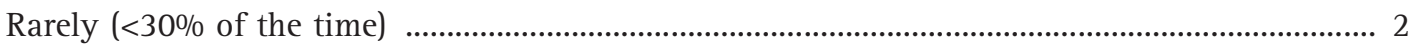

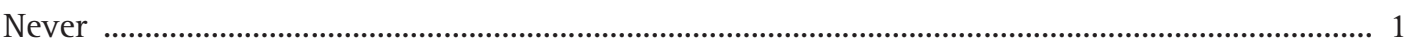

10. How often do you test for H. pylori in patients who are already on an NSAID?

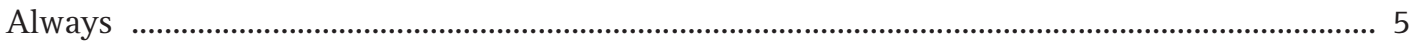

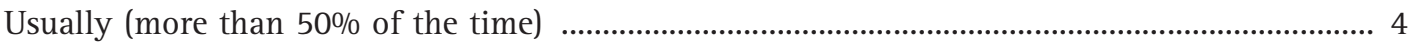

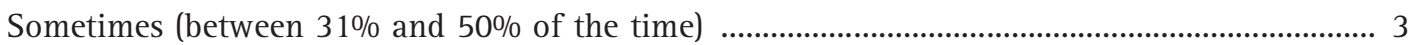

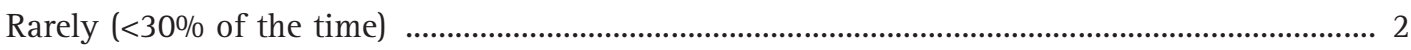

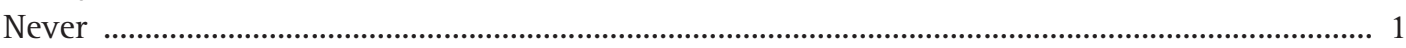

11. Thinking about when you prescribe NSAIDs, what percent of the NSAID prescriptions that you prescribe are traditional NSAIDs (e.g. ibuprofen) and what percent are COX-2-selective agents (e.g. Celebrex, Vioxx or Bextra)?

Traditional NSAIDs $\%$

COX-2-selective agents $\%$

MUST EQUAL 100\%

\section{Attitudes and beliefs}

12. Which of the following do you believe to be true regarding NSAID use (including aspirin) in individuals over the age of 70 ?

Less than $30 \%$ use an NSAID at least once per week ................................................................. 1

Between 30\% and 50\% use an NSAID at least once per week .................................................. 2

More than 50\% use an NSAID at least once per week .......................................................... 3 
13. Do you feel that traditional NSAIDs are associated with an increased risk of bleeding from lesions in the small intestine and/or colon?

Yes

No 2

14. Which statement do you feel best characterizes the relationship between NSAIDs and H. pylori?

The presence of $H$. pylori reduces the likelihood of ulcers in NSAID users ................................. 1

The presence of $H$. pylori has no effect on the likelihood of ulcers in NSAID users ................... 2

The presence of $H$. pylori increases the likelihood of ulcers in NSAID users 3

15. For each of the medications you see listed below, please indicate how likely you feel each is to cause ulcers and complications such as bleeding

\begin{tabular}{|c|c|c|c|c|c|c|c|c|c|c|}
\hline & Extremely likely 10 & 9 & 8 & 7 & 6 & 5 & 4 & 3 & 2 & Not at all likely 1 \\
\hline $\begin{array}{l}\text { Vioxx (rofecoxib) } \\
\text { Celebrex (celecoxib) } \\
\text { Naproxen } \\
\text { Ibuprofen } \\
\text { Voltaren (diclofenac) }\end{array}$ & & & & & & & & & & \\
\hline
\end{tabular}

16. How do you feel the use of low-dose aspirin influences the GI safety benefit of the COX-2-selective agents? Would you say that low-dose aspirin:

Increases the GI safety benefit 1

Decreases the GI safety benefit 2

Has no effect on the GI safety benefit 3

17. How much do you associate Vioxx (rofecoxib) with a higher likelihood of MI compared to other COX-2-selective agents? Please use a scale of 1-10 where a 10 means that the likelihood is significantly higher and 1 means that there is no difference in the likelihood of MI compared with other COX-2-selective agents.

Significantly more likely $10 \quad \begin{array}{llllllllll}10 & 9 & 7 & 6 & 5 & 4 & 3 & 2 & \text { No more likely than other COX-2-selective agents } 1\end{array}$

18. How much influence does concern about potential cardiac effects have on your decision to prescribe Vioxx (rofecoxib)? Please use a scale of 1-10 where a 10 means that it is extremely influential and 1 means that it does not influence you at all.

$\begin{array}{lllllllllll}\text { Extremely influential } 10 & 9 & 8 & 7 & 6 & 5 & 4 & 3 & 2 & \text { No influence at all } 1\end{array}$

19. For the next question we have listed several statements. Please read each statement carefully and indicate whether you feel that statement is true or false. 
The majority of patients who develop an upper gastrointestinal bleed as a consequence of an NSAID-related ulcer have experienced antecedent dyspeptic symptoms.

Low-dose aspirin has been shown to be effective in the primary (no previous history of infarction) prevention of MI.

Low-dose aspirin has been shown to be effective in the primary prevention of stroke.

Enteric coating or buffering of aspirin reduces the likelihood of developing a serious upper GI-bleeding event.

Compared with traditional NSAIDs, COX-2-selective agents reduce the likelihood of significant adverse GI events such as bleeding, perforation, or obstruction.

The likelihood of significant adverse GI events such as bleeding, perforation, or obstruction is reduced in a patient taking a COX-2-selective agents plus low-dose aspirin compared to a patient taking a traditional NSAID plus low-dose aspirin.

\section{APPENDIX}

Terminology

\section{Definition}

Traditional

NSAIDs

Proton pump

inhibitor (PPI)

H2RA

COX-2-selective

agents

NSAID
Non-steroidal anti-inflammatory drugs (e.g. Ibuprofen)

PPI (e.g. Prevacid)

Histamine-2-receptor sntagonist (e.g. Zantac)

A class of NSAIDs also referred to as COX-2 inhibitors (e.g. Vioxx)

For purposes of this research the general term NSAID refers to both traditional NSAIDs and cOX-2-selective agents. 